\title{
Labeling planar graphs with a condition at distance two ${ }^{\dagger}$
}

\author{
Peter Bella ${ }^{1}$, Daniel Král' ${ }^{23}$, Bojan Mohar ${ }^{4 \dagger}$ and Katarína Quittnerová ${ }^{1}$ \\ ${ }^{1}$ Department of Mathematical Analysis, Faculty of Mathematics and Physics, Charles University, Sokolovska 83, \\ 18675 Prague, Czech Republic. E-mail: XXYY@karlin.mff.cuni.cz. \\ ${ }^{2}$ Department of Applied Mathematics, Faculty of Mathematics and Physics, Charles University, Malostranské náměstí 25, \\ 11800 Prague, Czech Republic. E-mail: kral@kam.mff.cuni.cz. \\ ${ }^{3}$ Institute for Mathematics, Technical University Berlin, Strasse des 17. Juni 136, D-10623 Berlin, Germany. ${ }^{\S}$ \\ ${ }^{4}$ Department of Mathematics, University of Ljubljana, Jadranska 19, 1000 Ljubljana, Slovenia. \\ E-mail: bojan.mohar@fmf.uni-lj.si.
}

An $L(2,1)$-labeling of a graph is a mapping $c: V(G) \rightarrow\{0, \ldots, K\}$ such that the labels assigned to neighboring vertices differ by at least 2 and the labels of vertices at distance two are different. Griggs and Yeh [SIAM J. Discrete Math. 5 (1992), 586-595] conjectured that every graph $G$ with maximum degree $\Delta$ has an $L(2,1)$-labeling with $K \leq \Delta^{2}$. We verify the conjecture for planar graphs with maximum degree $\Delta \neq 3$.

Keywords: $L(2,1)$-labeling, channel assignment problem, graph coloring, planar graphs

\section{Contents}

\section{Introduction}

An $L(p, q)$-labeling is a popular graph theoretic model for the channel assignment problem. A vertexlabeling by non-negative integers of a graph $G$ is called an $L(p, q)$-labeling if the labels of neighboring vertices differ by at least $p$ and the labels of vertices at distance two differ by at least $q$. The least integer $K$ for which there exists such a labeling by integers between 0 and $K$ is denoted by $\lambda_{p, q}(G)$. Griggs

\footnotetext{
${ }^{\dagger}$ This research was conducted as a part of the Czech-Slovenian bilateral project MŠMT-07-0405 (Czech side) and SLO-CZ/0405-002 (Slovenian side).

${ }^{\ddagger}$ Supported in part by the the Ministry of Higher Education, Science and Technology of Slovenia, Research Project J1-05020101-00 and Research Program P1-0297.

$\S$ The author is a postdoctoral fellow at TU Berlin within the framework of the European training network COMBSTRU.

E-mail: kral@math.tu-berlin.de.
} 
and Yeh [9] conjectured that $\lambda_{2,1}(G) \leq \Delta^{2}$ for every graph $G$ with maximum degree $\Delta \geq 2$. The conjecture has been verified only for several classes of graphs such as graphs of maximum degree two, chordal graphs [19] (see also [4, 15]) and Hamiltonian cubic graphs [11, 12]. For general graphs, the original bound $\lambda_{2,1}(G) \leq \Delta^{2}+2 \Delta$ of [9] was improved to $\lambda_{2,1}(G) \leq \Delta^{2}+\Delta$ in [5]. A more general result of [14] yields $\lambda_{2,1}(G) \leq \Delta^{2}+\Delta-1$ and the present record of $\Delta^{2}+\Delta-2$ was proven by Gonçalves [8]. The algorithmic aspects of $L(2,1)$-labelings and $L(p, q)$-labelings in general have also been investigated $[1,2,6,7,13,16]$ because of their potential applications in practice.

Let us briefly survey known results on $L(p, q)$-labelings of planar graphs: van den Heuvel et al. [10] showed that $\lambda_{p, q}(G) \leq(4 q-2) \Delta+10 p-38 q-23$, Borodin et al. [3] provides the bound of $\lambda_{p, q}(G) \leq$ $(2 q-1)\lceil 9 \Delta / 5\rceil+8 p-8 q+1$ for $\Delta \geq 47$ and the best asymptotic result $\lambda_{p, q}(G) \leq q\lceil 5 \Delta / 3\rceil+18 p+$ $77 q-18$ is due to Molloy and Salavatipour [17, 18]. The bound of van den Heuvel et al. [10] implies that the conjecture of Griggs and Yeh holds for planar graphs with maximum degree $\Delta \geq 7$. We show that the conjecture holds for planar graphs with maximum degree $\Delta \neq 3$. The problem is treated in a more general setting of list labeling. Each vertex $v$ of a given graph $G$ is assigned a list of $k$ integers and it is required that the label of $v$ belongs its list. The smallest integer $k$ for which a proper $L(p, q)$-labeling exists for any list assignment with list sizes $k$ is denoted by $\operatorname{ch}_{p, q}(G)$.

\section{Our results}

Our approach is based on Discharging Method: first, reducible configurations, i.e., configurations that cannot appear in a minimal counterexample, are identified. Then, a minimal counterexample is considered and each vertex and face of it is assigned some charge. We always assign charge of $\operatorname{deg}(\alpha)-4$ to a vertex (face) $\alpha$ of degree $\operatorname{deg}(\alpha)$. The sum of the amounts of initial charge is -8 . In the final phase, the charge is redistributed by a certain set of rules and it is shown that the final amount of charge of each vertex and face is non-negative-contradiction.

Proving results on $L(2,1)$-labeling is more difficult than for the ordinary colorings, e.g., it cannot be assumed that minimal counterexamples are vertex 2-connected (or even bridgeless) or do not contain short cycles. In order to cope with this, we develop a notion of weak configuration: a weak configuration $G^{\prime}$ is a plane graph together with a mapping $d: V\left(G^{\prime}\right) \rightarrow \mathbb{N}$. $G^{\prime}$ appears in a plane graph $G$ if there is a mapping $\varphi: V\left(G^{\prime}\right) \rightarrow V(G)$ that preserves local structure of $G^{\prime}$ and $\operatorname{deg}_{G}(\varphi(v))=d(v)$ for all $v \in V\left(G^{\prime}\right)$. More precisely, for every vertex $v \in V\left(G^{\prime}\right)$ with neighbors $w_{1}, \ldots, w_{k}$ in $G^{\prime}, \varphi(v)$ is adjacent to $\varphi\left(w_{1}\right), \ldots, \varphi\left(w_{k}\right)$ in $G$ and the cyclic order of the vertices around $\varphi(v)$ is preserved. Similarly, the structure of faces of $G^{\prime}$ is preserved. The mapping $\varphi$ is not required to be injective (a single vertex of $G$ can correspond to several distinct vertices of $G^{\prime}$ ). In addition, the images of vertices of $G^{\prime}$ may be adjacent in $G$ even if they are not adjacent in $G^{\prime}$ (if the degree constraints allow incidences with edges not contained in $G^{\prime}$ ).

Lemma 1 Let $G^{\prime}$ with $d: V\left(G^{\prime}\right) \rightarrow \mathbb{N}$ be a weak configuration with $V\left(G^{\prime}\right)=\left\{v_{1}, \ldots, v_{n}\right\}$ and $K$ a positive integer. Assume that the following holds for every $i=1, \ldots, n$ :

$$
\begin{aligned}
6\left(d\left(v_{i}\right)-\operatorname{deg}_{G^{\prime}}\left(v_{i}\right)\right)+\sum_{v_{i^{\prime}} \in N_{G^{\prime}, 1}\left(v_{i}\right)}\left(d\left(v_{i^{\prime}}\right)-\operatorname{deg}_{G^{\prime}}\left(v_{i^{\prime}}\right)\right)+ & \\
& \operatorname{deg}_{G^{\prime}}\left(v_{i}\right)+\left|\left\{v_{1}, \ldots, v_{i-1}\right\} \cap N_{G^{\prime}, 2}\left(v_{i}\right)\right|<K .
\end{aligned}
$$

If $G^{\prime}$ appears in $G$ (via a mapping $\varphi$ ) and there is an an $L(2,1)$-labeling of $G \backslash \varphi^{-1}\left(V\left(G^{\prime}\right)\right)$ with span 
$K$ such that any two vertices of $G \backslash \varphi^{-1}\left(V\left(G^{\prime}\right)\right)$ whose distance in $G$ is two have different labels, then $\lambda_{2,1}(G) \leq K$.

Since we do not use planarity of $G$ in the proof of Lemma 1, we believe that the lemma and its proof may be found useful when coping with the conjecture for general graphs. Lemma 1 also makes it easy to verify whether a weak configuration is reducible using a computer (it is enough to find an appropriate ordering of the vertices and check the simple inequality from the statement).

As discussed in Section 1, the conjecture of Griggs and Yeh was not known to hold for planar graphs of maximum degree $\Delta \in\{3,4,5,6\}$. The case of $\Delta=6$ is dealt using eight reducible configurations and four rules are used in the discharging phase:

Theorem 1 If $G$ is a planar graph of maximum degree $\Delta=6$, then $\operatorname{ch}_{2,1}(G) \leq 33$. In particular, $\lambda_{2,1}(G) \leq 32$.

In the case of $\Delta=5$, we identify ten reducible configurations and use five rules in the discharging phase:

Theorem 2 If $G$ is a planar graph of maximum degree $\Delta=5$, then $\operatorname{ch}_{2,1}(G) \leq 26$. In particular, $\lambda_{2,1}(G) \leq 25$.

The case of $\Delta=4$ is more difficult. We identified 18 reducible configurations and the discharging phase involves 17 discharging rules. However, the analysis of the final charge of faces of degree five and six is computer-assisted: using a computer program, additional 1381 reducible configurations involving faces of degree five and additional 986 configurations involving faces of degree six were found. We have also been able to analyze the final charge of faces of degree six without the assistance of the computer. More details will be provided in the journal version of our paper and all the reducible configurations will be available on the web.

Theorem 3 If $G$ is a planar graph of maximum degree $\Delta=4$, then $\operatorname{ch}_{2,1}(G) \leq 17$. In particular, $\lambda_{2,1}(G) \leq 16$.

\section{References}

[1] G. Agnarsson, R. Greenlaw, M. M. Halldórsson, Powers of chordal graphs and their coloring, to appear in Congr. Numer.

[2] H. L. Bodlaender, T. Kloks, R. B. TAn, J. VAn LeEuWen, $\lambda$-coloring of graphs, G. Goos, J. Hartmanis, J. van Leeuwen, eds., Proc. STACS'00, LNCS Vol. 1770, Springer, 2000, 395-406.

[3] O. Borodin, H. J. Broersma, A. Glebov, J. VAn den Heuvel, Stars and bunches in planar graphs. Part II: General planar graphs and colourings, CDAM Reserach Report 2002-05, 2002.

[4] G. J. Chang, W.-T. Ke, D. D.-F. LiU, R. K. YeH, On L(d,1)-labellings of graphs, Discrete Math. 3(1) (2000), 57-66.

[5] G. J. Chang, D. Kuo, The L(2,1)-labeling problem on graphs, SIAM J. Discrete Math. 9(2) (1996), 309-316. 
[6] J. Fiala, J. Kratochvíl, T. Kloks, Fixed-parameter complexity of $\lambda$-labelings, Discrete Appl. Math. 113(1) (2001), 59-72.

[7] D. A. Fotakis, S. E. Nikoletseas, V. G. Papadopoulou, P. G. Spirakis, NP-Completeness results and efficient approximations for radiocoloring in planar graphs, B. Rovan, ed., Proc. MFCS'00, LNCS Vol. 1893, Springer, 2000, 363-372.

[8] D. GonçALVES, private communication.

[9] J. R. GRIGGS, R. K. YEH, Labeling graphs with a condition at distance 2, SIAM J. Discrete Math. 5 (1992), 586-595.

[10] J. VAN DEN Heuvel, S. MCGUINESS, Colouring of the square of a planar graph, J. Graph Theory 42 (2003), 110-124.

[11] J.-H. KANG, L(2, 1)-labeling of 3-regular Hamiltonian graphs, submitted for publication.

[12] J.-H. KANG, L(2, 1)-labelling of 3-regular Hamiltonian graphs, Ph.D. thesis, University of Illinois, Urbana-Champaign, IL, 2004.

[13] D. KRÁL', An exact algorithm for channel assignment problem, Discrete Appl. Math. 145(2) (2004), 326-331.

[14] D. KRÁL', R. ŠKREKOVSKI, A theorem about channel assignment problem, SIAM J. Discrete Math., 16(3) (2003), 426-437.

[15] D. KRÁL', Coloring powers of chordal graphs, SIAM J. Discrete Math. 18(3) (2004), 451-461.

[16] C. MCDIARMID, On the span in channel assignment problems: bounds, computing and counting, Discrete Math. 266 (2003), 387-397.

[17] M. Molloy, M. R. Salavatipour, A bound on the chromatic number of the square of a planar graph, to appear in J. Combin. Theory Ser. B.

[18] M. Molloy, M. R. Salavatipour, Frequency channel assignment on planar networks, R. H. Möhring, R. Raman, eds., Proc. ESA’02, LNCS Vol. 2461, Springer, 2002, 736-747.

[19] D. SAKAI, Labeling chordal graphs: distance two condition, SIAM J. Discrete Math. 7 (1994), $133-140$. 